\title{
Fork Tensor-Product States: Efficient Multiorbital Real-Time DMFT Solver
}

\author{
Daniel Bauernfeind, ${ }^{1, *}$ Manuel Zingl, ${ }^{1}$ Robert Triebl, ${ }^{1}$ Markus Aichhorn, ${ }^{1}$ and Hans Gerd Evertz ${ }^{1,2}$ \\ ${ }^{1}$ Institute of Theoretical and Computational Physics, Graz University of Technology, 8010 Graz, Austria \\ ${ }^{2}$ Kavli Institute for Theoretical Physics, University of California, Santa Barbara, California 93106, USA \\ (Received 23 December 2016; revised manuscript received 14 April 2017; published 20 July 2017) \\ We present a tensor network especially suited for multi-orbital Anderson impurity models and as an \\ impurity solver for multi-orbital dynamical mean-field theory (DMFT). The solver works directly on the \\ real-frequency axis and yields high spectral resolution at all frequencies. We use a large number $(\mathcal{O}(100))$ \\ of bath sites and therefore achieve an accurate representation of the bath. The solver can treat full \\ rotationally invariant interactions with reasonable numerical effort. We show the efficiency and accuracy of \\ the method by a benchmark for the three-orbital test-bed material $\mathrm{SrVO}_{3}$. There we observe multiplet \\ structures in the high-energy spectrum, which are almost impossible to resolve by other multi-orbital \\ methods. The resulting structure of the Hubbard bands can be described as a broadened atomic spectrum \\ with rescaled interaction parameters. Additional features emerge when $U$ is increased. Finally, we show \\ that our solver can be applied even to models with five orbitals. This impurity solver offers a new route to \\ the calculation of precise real-frequency spectral functions of correlated materials.
}

DOI: 10.1103/PhysRevX.7.031013

\section{INTRODUCTION}

Strongly correlated systems are among the most fascinating objects that solid-state physics has to offer. The interactions between constituents of such systems lead to emergent phenomena that cannot be deduced from the properties of noninteracting particles [1].

One of the most widely used methods to describe strongly correlated electrons is the dynamical mean-field theory (DMFT) [2,3]. DMFT treats local electronic correlations using a self-consistent mapping of the lattice problem onto an effective Anderson impurity model (AIM). Calculating the single-particle spectral function of this impurity model in an accurate and efficient way is at the heart of every DMFT calculation. To this end, many numerical methods have been developed or adapted. These are based, for instance, on continuous-time quantum Monte Carlo (CTQMC) [4,5], exact diagonalization (ED) [6-8], the numerical renormalization group (NRG) $[9,10]$, the configuration interaction (CI) [11,12], and also the density-matrix renormalization group (DMRG) with matrix-product states (MPS) $[13,14]$.

Every algorithm has strengths and weaknesses: CTQMC is exact, apart from statistical errors on the imaginary axis, and can deal with multiple orbitals, but it is, in some cases, plagued by the fermionic sign problem. Additionally, an ill-posed analytic continuation is necessary to obtain

\section{*daniel.bauernfeind@tugraz.at}

Published by the American Physical Society under the terms of the Creative Commons Attribution 4.0 International license. Further distribution of this work must maintain attribution to the author(s) and the published article's title, journal citation, and DOI.
Subject Areas: Condensed Matter Physics

real-frequency spectra, which therefore become broadened, especially at high energies. ED directly provides spectra on the real axis, but it is severely limited in the size of the Hilbert space, i.e., in the number of bath sites. Quite recently, NRG was shown to be a viable three-band solver by exploiting non-Abelian quantum number conservation [15-17]. NRG works on the real axis and captures the lowenergy physics well, but by construction, it has a poor resolution at higher energies. Another interesting route that has been proposed recently is the one of solvers that tackle the problem of exponential growth of the Hilbert space using ideas from quantum chemistry, i.e., the configuration interaction [11,12]. They allow one to go beyond the small bath sizes of ED, keeping all the advantages, such as the absence of fermionic sign problems. However, in multiorbital applications (see the Appendix of Ref. [12]), the spectral resolution has so far been restricted by the number of bath sites $[\mathcal{O}(20)]$.

Finally, MPS-based techniques like DMRG do not suffer from a sign problem and can be used on the real-frequency axis as well as on the imaginary-frequency axis. The absence of the sign problem, in general, comes at the cost of a very large growth of bond dimension with the number of orbitals.

Dynamical properties and spectral functions can be calculated within DMRG and have been used for impurity solvers, e.g., with the Lanczos-like continued-fraction expansion $[18,19]$. Other solvers using the more stable correction vector [20] and dynamical DMRG (DDMRG) [21] methods were developed [22-25]. Both algorithms produce very accurate spectral functions but have the disadvantage that a separate calculation for each frequency has to be performed. The Chebyshev expansion [26] with MPS [27], supplemented by linear prediction [28], was used for impurity solvers in the single-band case [29] and 
for two bands [30]. Recently, some of us introduced a method based on real-time evolution [31-34] and achieved a self-consistent DMFT solution for a two-band model [35]. In such calculations, the physical orbitals for each spin direction are usually combined into one large site in the MPS. Three or more orbitals have not been feasible with this approach because of a large increase in computational cost with the number of orbitals. Another MPS-based solver, which works on the imaginary axis, was recently introduced [36], and it was applied as a solver for three bands in two-site cluster DMFT. It was supplemented by a single real-time evolution to compute the spectral function, avoiding the analytic continuation. However, this method is restricted by the number of bath sites that can be employed. In the calculation mentioned, only three bath sites per orbital were used, limiting the energy resolution for realfrequency spectral functions.

In the present paper, we introduce a novel impurity solver that works directly on the real-frequency axis. To this end, we use a tensor network that captures the geometry of the interactions in the Anderson model better than a standard MPS. Our approach is, to some extent, inspired by the work of Ref. [37], which used a similar network for a two-orbital NRG ground-state calculation. We are not restricted to a small number of bath sites. This is imperative for exploiting the spectral resolution achievable with real-time calculations. We emphasize that (i) our method is, by construction, free of any fermionic sign problem, (ii) one can fully converge the DMFT self-consistency loop on the real-frequency axis, and (iii) we can achieve an almost exact representation of the bath spectral function. We apply this method to multi-orbital DMFT for the test-bed material $\mathrm{SrVO}_{3}$ and show that one can resolve a multiplet structure in the Hubbard bands, at the same time keeping a good description of the low-energy quasiparticle excitations.

The paper is structured as follows. First, we outline how impurity solvers with tensor networks work and introduce our new tensor network approach, which we call fork tensor-product states (FTPS) (Sec. II). Next, we explain in detail how our solver is used in the context of multi-orbital DMFT (Sec. III). In Sec. IV, we apply our approach to $\mathrm{SrVO}_{3}$ and discuss the multiplet structure that the FTPS solver allows us to resolve. In order to check the accuracy of the method, we also compare the FTPS results to CTQMC for $\mathrm{SrVO}_{3}$. Finally, we show the efficiency of the FTPS solver by applying it to a five-orbital model.

\section{TENSOR NETWORK IMPURITY SOLVERS}

The AIM describes an impurity (with Hamiltonian $H_{\text {loc }}$ ) coupled to a bath of noninteracting fermions hybridized with it. A typical AIM Hamiltonian is given by

$$
\begin{aligned}
H & =H_{\mathrm{loc}}+H_{\mathrm{bath}}, \\
H_{\mathrm{loc}} & =\epsilon_{0} \sum_{m \sigma} n_{m 0 \sigma}+H_{\mathrm{DD}}+H_{\mathrm{SF}-\mathrm{PH}}, \\
H_{\mathrm{DD}} & =U \sum_{m} n_{m 0 \uparrow} n_{m 0 \downarrow}+(U-2 J) \sum_{m^{\prime}>m, \sigma} n_{m 0 \sigma} n_{m^{\prime} 0 \bar{\sigma}}+(U-3 J) \sum_{m^{\prime}>m, \sigma} n_{m 0 \sigma} n_{m^{\prime} 0 \sigma}, \\
H_{\mathrm{SF}-\mathrm{PH}} & =J \sum_{m^{\prime}>m}\left(c_{m 0 \uparrow}^{\dagger} c_{m 0 \downarrow} c_{m^{\prime} 0 \uparrow} c_{m^{\prime} 0 \downarrow}^{\dagger}+\text { H.c. }\right)-J \sum_{m^{\prime}>m}\left(c_{m 0 \uparrow}^{\dagger} c_{m 0 \downarrow}^{\dagger} c_{m^{\prime} 0 \uparrow} c_{m^{\prime} 0 \downarrow}+\text { H.c. }\right), \\
H_{\text {bath }} & =\sum_{m l \sigma} \epsilon_{l} n_{m l \sigma}+V_{l}\left(c_{m 0 \sigma}^{\dagger} c_{m l \sigma}+\text { H.c. }\right),
\end{aligned}
$$

where $c_{m l \sigma}^{\dagger}\left(c_{m l \sigma}\right)$ creates (annihilates) an electron in band $m(m \in\{1,2,3\}$ for a three-orbital model) with spin $\sigma$ at the $l$ th site of the system (the impurity has index $l=0$; the bath degrees of freedom have $l \geq 1$ ), and $n_{m l \sigma}$ are the corresponding particle-number operators. Note that $H_{\mathrm{DD}}$ describes density-density (DD) interactions between all orbitals, and $H_{\mathrm{SF}-\mathrm{PH}}$ are the spin-flip and pair-hopping terms. This three-orbital Hamiltonian is not only important in the context of real-material calculations. It has also been studied extensively on the model level-most importantly, because it hosts unconventional correlation phenomena. For a selection of recent work, see, for instance, Refs. [15,38-41].

An impurity solver calculates the retarded impurity Green's function $G(t)$,

$$
G(t)=-i \theta(t)\left\langle\psi_{0}\left|\left[c^{\dagger}(t), c(0)\right]\right| \psi_{0}\right\rangle,
$$

of the interacting problem (1), either in real- or imaginarytime $t$. In the present paper, we introduce a new tensor network similar to a MPS, which can be used as a real-time impurity solver for three orbitals. We first introduce MPS before moving on to what we call fork tensor-product states in Sec. II B.

\section{A. MPS and DMRG}

MPS are a powerful tool to efficiently encode quantummechanical states. Consider a state $|\psi\rangle$ of a system consisting of $N$ sites: 


$$
|\psi\rangle=\sum_{s_{1}, s_{2}, \ldots, s_{N}} c_{s_{1}, \ldots, s_{N}}\left|s_{1}, s_{2}, \ldots, s_{N}\right\rangle .
$$

Each site $i$ has a local Hilbert space of dimension $d_{i}$ spanned by the states $\left|s_{i}\right\rangle$. Through repeated use of singular-value decompositions (SVDs), it is possible to factorize every coefficient $c_{s_{1}, \ldots, s_{N}}$ into a product of matrices [14], i.e., into a MPS,

$$
|\psi\rangle=\sum_{s_{1}, s_{2}, \ldots, s_{N}} A_{1}^{s_{1}} \cdot A_{2}^{s_{2}} \cdots A_{N}^{s_{N}}\left|s_{1}, s_{2}, \ldots, s_{N}\right\rangle .
$$

Each $A_{i}^{s_{i}}$ is a rank-three tensor, except the first and last ones $\left(A_{1}^{s_{1}}, A_{N}^{s_{N}}\right)$, which are of rank two. The index $s_{i}$ is called a physical index, and the matrix indices, which are summed over, are the so-called bond indices. A general state of the full Hilbert space is not feasible to store, but it can be shown that ground states are well described by a MPS with limited bond dimension $m$ (dimension of the bond index) [42].

In complete analogy to the states, one can factorize an operator into what is called a matrix-product operator (MPO) [14],

$$
H=\sum_{\substack{s_{1}, \ldots, s_{N} \\ s_{1}^{\prime} \ldots, s_{N}^{\prime}}} W_{1}^{s_{1}, s_{1}^{\prime}} \cdots W_{N}^{s_{N}, s_{N}^{\prime}}\left|s_{1}^{\prime}, \ldots, s_{N}^{\prime}\right\rangle\left\langle s_{1}, \ldots, s_{N}\right|,
$$

where each $W_{i}^{s_{i}, s_{i}^{\prime}}$ is a rank-four tensor. Tensor networks, in general, have a very useful graphical representation, which is shown for a MPS and a MPO in Fig. 1. Note that when we use the term MPS, we always mean a one-dimensional chain of tensors, as shown in Fig. 1.

To calculate Green's functions within the MPS formalism, one usually first applies the DMRG [13,14], which acts on the space of MPS and finds a variational ground

(a)

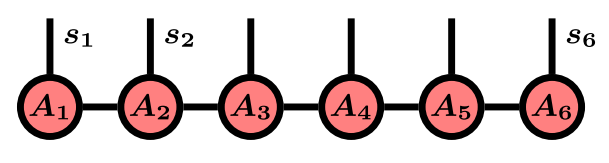

(b)

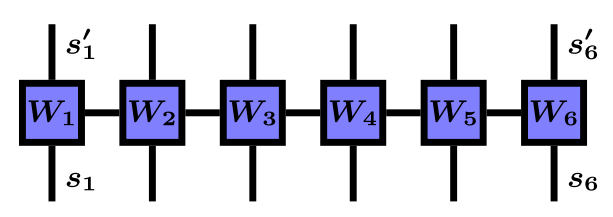

FIG. 1. (a) Graphical representation of a MPS. Every circle corresponds to a tensor $A_{i}^{s_{i}}$ and each line to an index of this tensor. In this picture, the physical indices are the vertical lines, while the horizontal lines show the bond indices. Connected lines mean that the corresponding index is summed over. Fixing all the physical indices $s_{i}$ for each site results in a tensor of rank zero with the value of the coefficient $c_{s_{1}, \ldots, s_{N}}$. (b) Graphical representation of a MPO. The difference from a MPS is that a MPO has incoming indices $s_{i}$ and outgoing indices $s_{i}^{\prime}$ corresponding to the bra- and ket vectors of the operator. state $\left|\psi_{0}\right\rangle$ and ground-state energy $E_{0}$. It minimizes the expectation value

$$
E_{0}=\min _{|\psi\rangle} \frac{\langle\psi|H| \psi\rangle}{\langle\psi \mid \psi\rangle},
$$

usually by updating two neighboring MPS tensors before moving on to the next bond. This procedure also yields the Schmidt decomposition of the state at the current bond on the fly. The DMRG approximation keeps only those states with the largest Schmidt coefficient. It is important to note that one can perform a DMRG calculation for any tensor network, as long as one can generate a Schmidt decomposition [43].

For obtaining the Green's function, we employ an evolution in real-time. Equation (2) is split into the greater $\left(G^{>}\right)$and lesser Green's function $\left(G^{<}\right)$:

$$
\begin{aligned}
G(t) & =-i \Theta(t)\left(G^{>}(t)+G^{<}(t)\right), \\
G^{>}(t) & =\left\langle\psi_{0}\left|c e^{-i H t} c^{\dagger}\right| \psi_{0}\right\rangle e^{i E_{0} t}, \\
G^{<}(t) & =\left\langle\psi_{0}\left|c^{\dagger} e^{i H t} c\right| \psi_{0}\right\rangle e^{-i E_{0} t},
\end{aligned}
$$

which are calculated in two separate time evolutions. This is done by first applying $c^{\dagger}$ (or $c$ ) and then time evolving this state and calculating the overlap with the state at time $t=0$. The time evolution is the most computationally expensive part since time-evolved states are not ground states anymore, and the needed bond dimensions usually grow very fast with time.

\section{B. Fork tensor-product states}

So far, the usual way of dealing with Hamiltonians like Eq. (1) using MPS $[29,30,35]$ has been to place the impurity in the middle of the system and the up- and down-spin degrees of freedom to its left and right, respectively. The local state space of each bath site then consists of $M$ spinless-fermion degrees of freedom, with dimension $2^{M}$, where $M$ is the number of orbitals in the Hamiltonian Eq. (1). This exponential growth is usually accompanied by a very fast growth in bond dimension when using the above arrangement. We did indeed encounter this very fast growth upon calculating the ground state of some one-, two-, and three-orbital test cases.

For treatment by MPS, the general Hamiltonian Eq. (1) with hopping terms from the impurity to each bath site is usually transformed into a Wilson chain with nearestneighbor hoppings only, i.e., of the form $t_{i}\left(c_{i}^{\dagger} c_{i+1}+\right.$ H.c.) [10]. This was thought to be necessary since longrange interactions look problematic for MPS-based algorithms. Quite recently, though, it was discovered that MPS can deal with the original form of $H_{\text {bath }}$ in Eq. (1) better [44]. Because all hopping terms in $H_{\text {bath }}$ originate from the impurity, this is called the star geometry. The reason for the better performance is that in the star geometry, one has 
many nearly fully occupied (empty) bath sites with very low (high) on-site energies $\epsilon_{l}$.

Since basis states with many unoccupied low-energy sites have a very low Schmidt coefficient, these states are discarded from the MPS. The same holds for occupied high-energy sites. However, when dealing with multi-orbital models, the star geometry is not enough to be able to calculate Green's functions using MPS. The growth of the bond dimensions still makes those calculations unfeasible.

The key idea of the present work is to construct a tensor network which is beyond a standard MPS but similar enough to be able to use established methods like DMRG and time evolution. From Hamiltonian (1), one can immediately notice that there are no terms coupling bath sites of different orbitals. Hence, it might not be advantageous to combine those (not directly interacting) degrees of freedom into one large physical index in the MPS.

Therefore, our proposed tensor network separates the bath degrees of freedom as much as possible. It consists of separate tensors for every orbital-spin combination, each connected to bath tensors as shown in Fig. 2. This tensor network is not a MPS anymore since there are some tensors (labeled $A \downarrow$ and $B \uparrow$ in the example of Fig. 2) that have three bond indices and one physical index, i.e., which are of rank four. Cutting any bond splits the network into two separate parts. Therefore, one can calculate the Schmidt decomposition in a way very similar to a MPS, which means that DMRG is also possible. The main bottleneck of calculations with FTPS is to perform SVDs of the rank-four

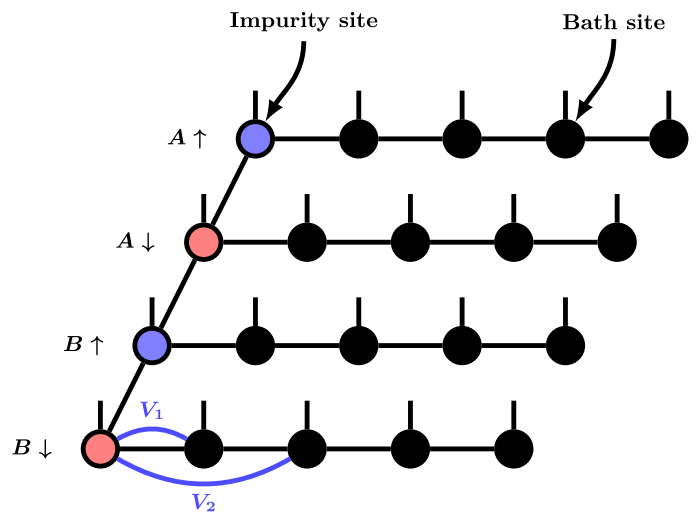

FIG. 2. Graphical representation of a FTPS for multi-orbital AIM. Separating bath degrees of freedom leads to a forklike structure. In this picture, a two-orbital model is shown, with four bath sites in each orbital. Orbitals are labeled $A$ and $B$, and the arrows denote the spin. Each spin-orbital combination has its own bath sticking out to the right. As in Fig. 1, the vertical lines are the physical degrees of freedom [all of dimension two, for empty (respectively, occupied) bath sites]. All other lines are bond indices, and like in the MPS, they are summed over. As mentioned in the text, the bath is represented in star geometry because of the smaller bond dimensions needed. The bath sites are ordered according to their on-site energies. Two example hoppings $V_{1}$ and $V_{2}$ are drawn. tensors representing the impurities. When all bond indices have the same dimension $\chi$, it is necessary to do a SVD for a $\chi^{2} d \times \chi$ matrix with computational complexity $\mathcal{O}\left(\chi^{4} d\right)$. However, as we show below, this operation does not pose a substantial problem for calculations using FTPS. Since the impurity tensors pose the biggest challenge, our tensor network would likely also allow us to deal with the chain geometry without a drastic increase in computational cost. In the present paper, we only use FTPS with baths in star geometry. The proposed FTPS are similar to the tensor network used by Holzner et al. [37] to perform NRG calculations for ground-state properties of an AIM with two orbitals.

The three-legged tensors in our network (Fig. 2) can also be interpreted as two coupled junctions with three legs in the language of Ref. [45], where it has been shown that DMRG is possible on such junctions. Furthermore, our approach has similarities to the so-called tree tensor networks (TTN) [43,46-48].

\section{Time evolution}

Time evolution with the Hamiltonian Eq. (1) is not straightforward since it features long-range hoppings. Possible methods include Krylov approaches [49], the time-dependent variational principle [50,51], and the series expansion of $e^{i H t}$ proposed by Zaletel et al. [52]. In this work, however, we use a much simpler approach.

First, we split the Hamiltonian into the following terms: (i) the spin-flip and pair-hopping terms $H_{m, m^{\prime}}^{\mathrm{SF}-\mathrm{PH}}$ for each orbital combination, with $\sum_{m^{\prime}>m} H_{m, m^{\prime}}^{\mathrm{SF}} \stackrel{\mathrm{PH}}{=} H_{\mathrm{SF}-\mathrm{PH}}$ [see Eq. (1)], (ii) the density-density interaction terms $H_{\mathrm{DD}}$, and (iii) all other terms, $H_{\text {free }}=H_{\text {bath }}+\epsilon_{0} \sum_{m \sigma} n_{m 0 \sigma}$. With these terms, we write the time-evolution operator for a small time step $\Delta t$ using a second-order Suzuki-Trotter decomposition [53],

$$
\begin{aligned}
e^{-i \Delta t H} \approx & \left(\prod_{m^{\prime}>m} e^{-i[(\Delta t) / 2] H_{m, m^{\prime}}^{\mathrm{SF}-\mathrm{PH}}}\right) e^{-i[(\Delta t) / 2] H_{\mathrm{DD}}} . \\
& \times e^{-i \Delta t H_{\text {free }}} e^{-i[(\Delta t) / 2] H_{\mathrm{DD}}}\left(\prod_{m^{\prime}>m} e^{-i[(\Delta t) / 2] H_{m, m^{\prime}}^{\mathrm{SF}-\mathrm{PH}}}\right)
\end{aligned}
$$

Note that in this decomposition, the order of the spin-flip and pair-hopping terms is important. The order of operators in the second product must be opposite to the one in the first.

We see that Eq. (8) involves three different operators, $H_{m, m^{\prime}}^{\mathrm{SF}}, H_{\mathrm{DD}}$, and $H_{\text {free }}$, each of which will be treated differently.

Time evolution of the density-density interactions is performed with a MPO-like representation of the timeevolution operator $e^{-i[(\Delta t) / 2] H_{\mathrm{DD}}}$. For a three-orbital model, first the full matrix $\left(4^{3} \times 4^{3}\right)$ of $e^{-i[(\Delta t) / 2] H_{D D}}$ is created, which is then decomposed into MPO form by repeated SVDs. Since $H_{\mathrm{DD}}$ only consists of density-density interactions, no fermionic sign appears in $e^{-i \Delta t H_{\mathrm{DD}}}$. 
Time evolution of the spin-flip and pair-hopping terms is more involved than the density-density interactions since the operators change the particle numbers on the impurity sites. Therefore, it can be difficult to deal with the fermionic sign of the time-evolution operator when the impurities are not next to each other in the fermionic order. It turns out that the spin-flip and the pair-hopping terms have the property $\hat{A}^{3}=\hat{A}$ individually, with $\hat{A}$ being either the spinflip or the pair-hopping operator, respectively. Furthermore, they commute with each other, allowing us to separate them without Trotter error. The time-evolution operator of $J \hat{A}$ is then given by

$$
e^{-i \Delta t J \hat{A}}=\mathbb{1}+\hat{A}^{2}(\cos (\Delta t J)-1)-i \hat{A} \sin (\Delta t J) .
$$

For this operator, a MPO can be found for which the fermionic sign can easily be determined [54].

To time evolve the bath terms, we use an iterative secondorder Suzuki-Trotter breakup for each term in $H_{\text {free- }}$. Neglecting orbital $(m)$ and spin $(\sigma)$ indices, the first step in this breakup is the following: $e^{-i \Delta t \sum_{l=1}^{N_{b}} H_{l}} \approx$ $e^{-i[(\Delta t) / 2] H_{1}} e^{-i \Delta t \sum_{l=2}^{N_{b}} H_{l}} e^{-i[(\Delta t) / 2] H_{1}}$. Next, we split off $H_{2}$ and iterate this procedure until we end up with

$$
\begin{aligned}
e^{-i \Delta t H_{\text {free }}} \approx & \prod_{m \sigma}\left[\left(\prod_{l=1}^{N_{b}-1} e^{-i \frac{\Delta t}{2} H_{m l \sigma}}\right)\right. \\
& \left.\times e^{-i \Delta t H_{m N_{b} \sigma}}\left(\prod_{l=N_{b}-1}^{1} e^{-i \frac{\Delta t}{2} H_{m l \sigma}}\right)\right],
\end{aligned}
$$

with $N_{b}$ the number of bath sites and $H_{m l \sigma}=$ $\epsilon_{l} n_{m l \sigma}+V_{l}\left(c_{m 0 \sigma}^{\dagger} c_{m l \sigma}+\right.$ H.c. $)$. In the above equation, we neglected the term $\epsilon_{0} n_{m 0 \sigma}$ that we add to $H_{m 1 \sigma}$. Equation (10) is a product of two-site gates (an operator acting nontrivially only on two sites) with one of the two sites always being the impurity. This means that those two sites are not nearest neighbors in the tensor network. To overcome this problem, we use so-called swap gates $[14,55]$. The two-site operator

$$
S_{i j}=\delta_{s_{i}, s_{j}^{\prime}} \delta_{s_{j}, s_{i}^{\prime}}(-1)^{n_{i} n_{j}}
$$

swaps the state of site $i$ ( $s_{i}$ with occupation $n_{i}$ ) with the state of site $j\left(s_{j}\right.$ with occupation $\left.n_{j}\right)$. The factor $(-1)^{n_{i} n_{j}}$ gives the correct fermionic sign and is negative if an odd number of particles on site $i$ gets swapped with an odd number of particles on site $j$. To be more precise, the matrix representation of the swap gates used in this work is

$$
S_{i j}=|00\rangle\langle 00|+| 10\rangle\langle 01+\mid 01\rangle\langle 10|-| 11\rangle\langle 11| .
$$

It turns out that every swap gate can be combined with an actual time-evolution gate without additional computational time. For example, the first step in this time evolution would be to

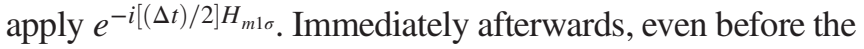

SVD (to separate the tensors again), the swap gate is applied so that the impurity and the first bath sites are swapped. By repeating this process, one moves the impurity along its horizontal arm in Fig. 2. Because a second-order decomposition is used, now all time-evolution gates except the one at site $N_{b}$ have to be applied again. But now, the impurity and bath sites need to be swapped before time evolution.

Note that not only can the algorithm presented above be used to perform real-time evolutions, but it is also applicable to evolution in imaginary-time simply by replacing $i d t$ by $d \tau$.

\section{MULTI-ORBITAL DMFT WITH FTPS}

In this section, we present details of our impurity solver. We refer to Refs. [2,56] for DMFT in general, and to Refs. $[57,58]$ for DMFT in the context of realistic ab initio calculations for correlated materials.

In the latter approach, called density-functional theory (DFT)+DMFT, the correlated subspace is described by a Hubbard-like Hamiltonian. Within DMFT, this model is mapped onto the AIM Hamiltonian (1). This mapping defines the bath hybridization function $\Delta(\omega)$ describing the influence of the surrounding electrons.

Since FTPS provide the Green's function of the AIM on the real-frequency axis, the self-consistency loop is also performed directly for real frequencies. For calculating the bath hybridization, we use retarded Green's functions with a finite broadening $\eta_{\mathrm{SC}}$ in order to avoid numerical difficulties with the poles of the Green's function. Throughout this work, we use $\eta_{\mathrm{SC}}=0.005 \mathrm{eV}$ [59].

The impurity solver calculates the self-energy $\Sigma(\omega)$ of the AIM, given the bath hybridization function $\Delta(\omega)$ and the interaction Hamiltonian on the impurity. To this end, our solver performs the following steps, which are explained in more detail in the text below:

(1) Obtain bath parameters $\epsilon_{l}$ and $V_{l}$ by a deterministic approach based on integration of the bath hybridization function $\Delta(\omega)$.

(2) Calculate the ground state $\left|\psi_{0}\right\rangle$ and ground-state energy $E_{0}$ of the interacting problem.

(3) Apply impurity creation or annihilation operators, and time evolve these states to determine the interacting Green's function [Eq. (2)].

(4) Fourier transform Eq. (2) to obtain $G(\omega)$ and calculate the local self-energy,

$$
\Sigma(\omega)=G_{0}(\omega)^{-1}-G(\omega)^{-1} .
$$

To perform step 1 we use

$$
\begin{aligned}
V_{l}^{2} & =\int_{I_{l}}\left[-\frac{1}{\pi} \operatorname{Im} \Delta(\omega)\right] d \omega, \\
\epsilon_{l} & =\frac{1}{V_{l}^{2}} \int_{I_{l}} \omega\left[-\frac{1}{\pi} \operatorname{Im} \Delta(\omega)\right] d \omega,
\end{aligned}
$$


similar to Refs. [10] (NRG) and [44]. Each interval $I_{l}$ corresponds to a bath site. This discretization can be interpreted as representing each interval $I_{l}$ as a delta peak at position $\epsilon_{l}$ and weight $V_{l}^{2}$. Sum rules for such discretization parameters can be found analytically [60]. In this work, we choose the length of each interval such that the area of the bath spectral function $-(1 / \pi) \operatorname{Im} \Delta(\omega)$ is approximately constant for each interval [61]. For the case at hand, this discretization was found to be numerically more stable than using intervals of constant length. Unless stated otherwise, we use $N_{b}=109$ bath sites per orbital and spin. We note that this scheme is not restricted to diagonal hybridizations. In the general case of off-diagonal hybridizations, the hybridization function is a matrix $\underline{\Delta}$. Therefore, instead of taking the imaginary part, we can use the bath spectral function $[i /(2 \pi)]\left(\underline{\Delta}-\underline{\Delta}^{\dagger}\right)$. Similarly to Eq. (14), we represent each interval by one delta peak for each orbital. For instance, fixing $\epsilon_{l}$ to the center of the interval, the hopping parameters $V_{l}$ can be found systematically from the Cholesky factorization of $\int_{I_{l}}[i /(2 \pi)]\left(\underline{\Delta}-\underline{\Delta}^{\dagger}\right) d \omega$. Most importantly, this scheme does not involve any fitting procedure on the Matsubara axis. A very similar approach was developed independently in Ref. [62].

In step 2, we use a DMRG approach with the following parameters, unless specified otherwise. The truncated weight $t_{w}$ (sum of all discarded singular values of each SVD) is kept smaller than $10^{-8}$. When spin-flip and pairhopping terms are neglected, we use an even smaller cutoff of $10^{-9}$. Note that, except in the five-band calculation, we do not restrict the bond dimensions by some hard cutoff (see Appendix A 2).

During time evolution (step 3), we use a truncated weight of $t_{w}=2 \times 10^{-8}$, or $10^{-8}$ with density-density interactions only. We time evolve to $t=16 \mathrm{eV}^{-1}$, with a time step of $\Delta t=0.01 \mathrm{eV}^{-1}$. Green's functions are measured every fifth time step. The time-evolution operator of $H_{\mathrm{loc}}$ is applied using the zip-up algorithm [55]. Afterwards, the Green's functions are extrapolated in time using the linearprediction method $[28,35]$ up to $t=250 \mathrm{eV}^{-1}$. Time evolution is split into two runs, one forward and one backwards in time [63], to be able to reach longer times.

In the Fourier transform to $\omega$ space (step 4), we use a broadening in the kernel $e^{i \omega t-\eta_{\mathrm{FT}}|t|}$ of $\eta_{\mathrm{FT}}=0.02 \mathrm{eV}$ to avoid cutoff effects remaining after the linear prediction. The influence of the linear prediction on our results is discussed in Appendix A 1. We stress that although a calculation with full rotational symmetry is more demanding, the computational effort is still very feasible. With the parameters mentioned above, one full DMFT cycle takes about five hours on 16 cores.

To verify that our implementation of DMRG and time evolution produces correct results when used with our tensor network, we first compared Green's functions and ground-state energies for $U=J=0$ for several bath parameter sets. The next step of our testing was to include density-density interactions, one term at a time. For example, we only included $\left(U^{\prime}-J\right) n_{10 \uparrow} n_{30 \uparrow}$ and compared energy and Green's functions to a standard one-orbital MPS solver. Finally, we also compared our method to the MPS two-band solver used in Ref. [35]. Indeed, all tests performed produced the correct energies and Green's functions.

\section{RESULTS}

We performed DMFT calculations based on a band structure obtained from DFT for the prototypical compound $\mathrm{SrVO}_{3}$, using the approximation of the Kanamori Hamiltonian [Eq. (1)]. It has a cubic crystal structure with a nominal filling of one electron in the V-3d shell [64]. Because of the crystal symmetry, the five orbitals of the V-3d shell split into two $e_{g}$ and three $t_{2 g}$ orbitals. The latter form the correlated subspace. We performed the DFT calculation with Wien2k [65] and used $34220 k$ points in the irreducible Brillouin zone in order to reach an energy resolution comparable with the $\eta_{\mathrm{SC}}=0.005 \mathrm{eV}$ broadening.

The TRIQS/DFTTools package (v1.4) [66-68], which is based on the TRIQS library (v1.4) [69], was used to generate the projective Wannier functions and to perform the DMFT self-consistency cycle.

Figure 3 shows the main results of this paper: the DMFT spectral function $A(\omega)$ for $\mathrm{SrVO}_{3}$, (i) in the approximation of density-density interactions only and (ii) with full rotational invariance, including spin-flip and pair-hopping terms. Overall, both cases show the well-known features of the $\mathrm{SrVO}_{3}$ spectral function [70,71]. We see a hole excitation at around $-2 \mathrm{eV}$, as well as the quasiparticle peak at zero energy whose shape and position does not

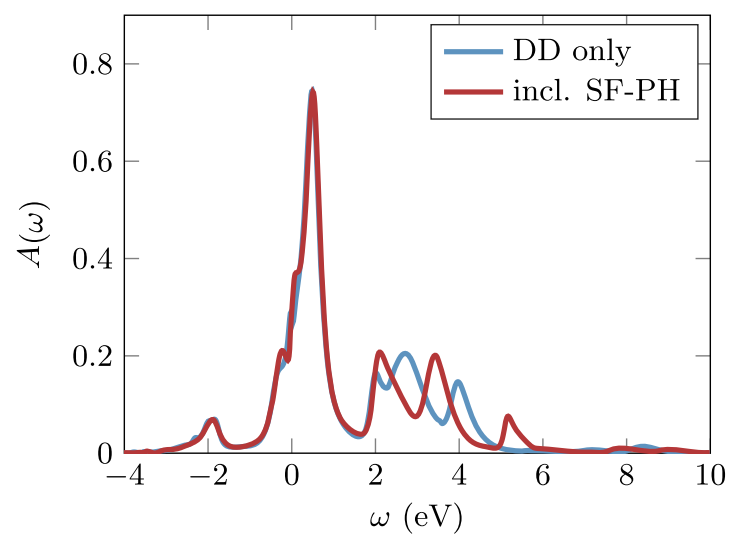

FIG. 3. Spectral functions $A(\omega)$ for density-density interactions (DD) only (blue line), and with spin-flip and pair-hopping terms included (red line). In both calculations, we used $U=$ $4.0 \mathrm{eV}$ and $J=0.6 \mathrm{eV}$. Both spectra show a three-peak structure in the upper Hubbard band and additional features at high energies (around $8 \mathrm{eV}$ ). 
depend on the inclusion of full rotational invariance. In the upper Hubbard band, a distinctive three-peak structure can be seen. This structure has not been resolved in other exact methods like CTQMC (because of problems with analytic continuation, see below) or NRG (because of the logarithmic discretization problem). In our real-time approach, high energies correspond to short times, where the calculations are particularly precise [72]. Most methods allow us to resolve structures in the Hubbard bands only in special cases (see Ref. [73] for an example using ED). Of course, atomic-limit-based algorithms such as the Hubbard-I approximation or noncrossing approximation (NCA) show atomiclike features, but they have very limited accuracy for the description of the low-energy quasiparticle excitations in the metallic phase [74]. Thus, our FTPS solver combines the best of both worlds, with atomic multiplets at high energy and excellent low-energy resolution at the same time.

The energies of the three peaks in the upper Hubbard band differ depending on whether SF-PP terms are taken into account or not. Details of this peak structure, as well as additional excitations visible at higher energies, will be discussed below in Sec. IV C.

First, we examine the convergence of our results with respect to the number of bath sites and compare our spectrum to CTQMC. The following discussion is mostly based on calculations without spin-flip and pair-hopping terms. In this case, the calculations can be done faster and with higher precision since there is no particle exchange between impurities. In all subsequent plots, we show results from calculations with DD interactions only.

\section{A. Effect of bath size}

In order to achieve a reliable high-resolution spectrum on the real-frequency axis, it is imperative to have a good representation of the hybridization function $\Delta(\omega)$ in terms of the bath parameters, for which a sufficient number of bath sites is needed. Figure 4 shows how well a hybridization function can be represented with our approach [Eq. (14)] using a certain number of bath sites. We see that for $N_{b}=9$ bath sites (we always denote sites per orbital), $\Delta(\omega)$ can be reconstructed only very roughly, which in turn gives an incorrect spectral function (Fig. 4, bottom). To some extent, the difference in the spectrum is due to the shorter time evolution and therefore a higher broadening, $\eta_{\mathrm{FT}}$, which we were forced to use. For such a small bath, the finite-size effects from reflections at the bath ends appear much earlier in the time evolution.

Increasing the number of bath sites to $N_{b}=29$, we observe that the reconstructed bath spectral function already shows the relevant features of $\Delta(\omega)$. The spectrum is well converged for the largest bath sizes $N_{b}=59$ and $N_{b}=109$.
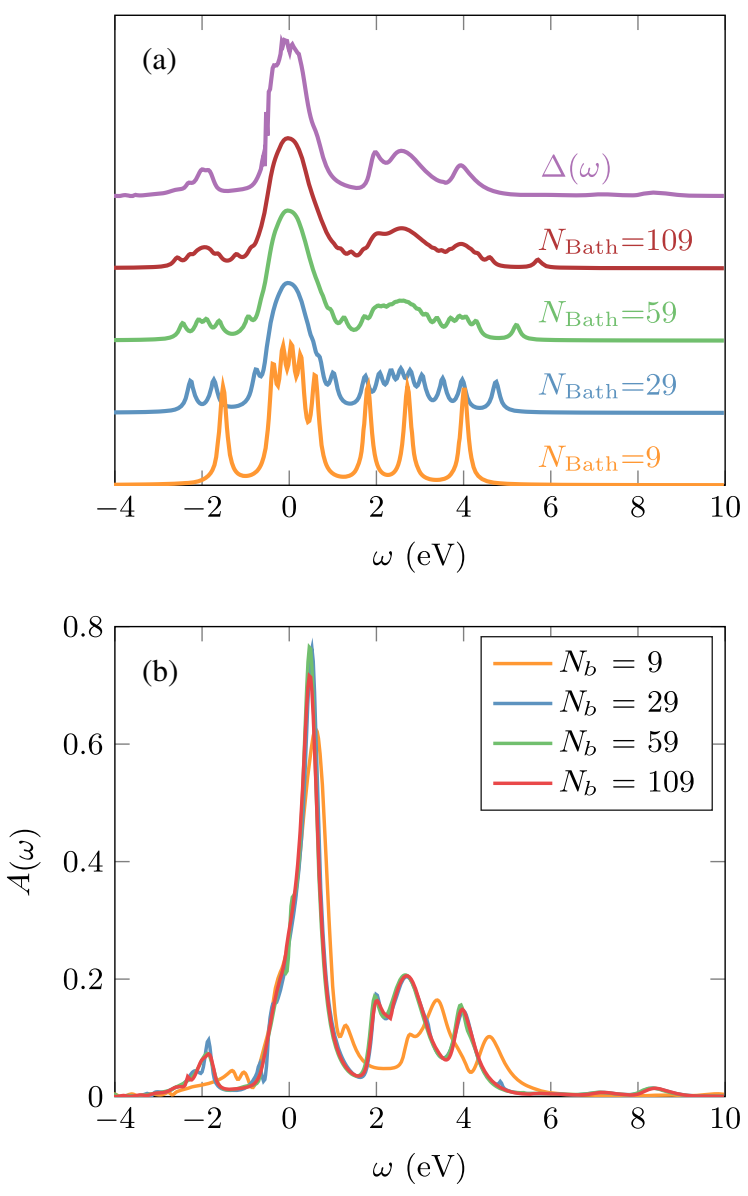

FIG. 4. (a) We take the bath spectral function $\Delta(\omega)$ from the DMFT self-consistent solution for $N_{b}=109$ and represent it using various numbers of bath sites. It is obvious that $N_{b}=9$ is too small to represent the bath well. (b) Converged DMFT spectral function using the AIM with different numbers of bath sites. Only the smallest bath shows a noticeable difference. This is mostly due to the fact that, in this case, a higher broadening of $\eta_{\mathrm{FT}}=0.1 \mathrm{eV}$ had to be used in the Fourier transform and time evolution was only possible for $t=14 \mathrm{eV}^{-1}$. The additional small structure at $\omega=0$ for $N_{b}=59$ bath sites is most likely a linear-prediction artifact.

\section{B. Comparison to CTQMC}

In Fig. 5, we compare the converged spectral function of our approach (FTPS) with a spectrum obtained from CTQMC and analytic continuation. In both calculations, we used the same interaction Hamiltonian with densitydensity interactions only. The CTQMC calculation was performed with the TRIQS CTHYB solver (v1.4) $[75,76]$ with $3.2 \times 10^{7}$ measurements and at inverse temperature $\beta=200 \mathrm{eV}^{-1}$. For the analytic continuation, we applied the $\Omega$ MaxEnt method [77].

The three-peak structure in the upper Hubbard band is not present in the CTQMC spectrum. We show in an example below that even for a Green's function that does contain these peaks, the analytic continuation does not resolve this structure. 


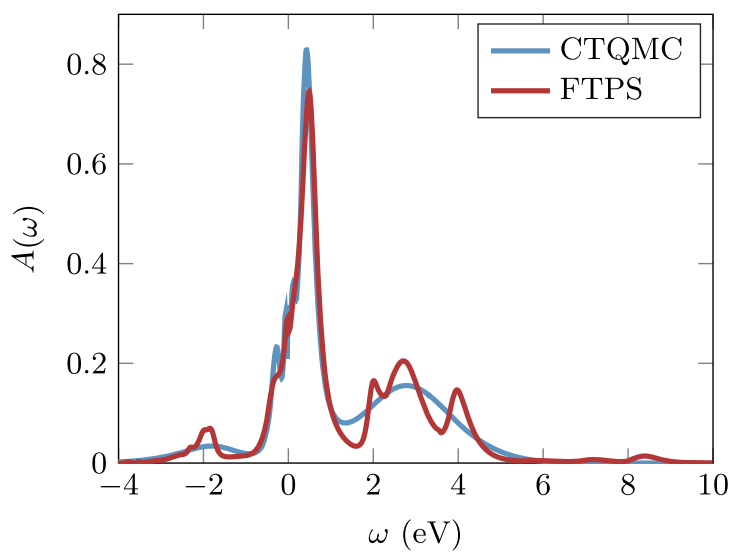

FIG. 5. DMFT spectral functions $A(\omega)$ from CTQMC + MaxEnt (blue line) at $\beta=200 \mathrm{eV}^{-1}$, and from FTPS (red line). The FTPS result shows a distinctive three-peak structure in the upper Hubbard band.

For another comparison, we consider the imaginary-time Green's functions $G(\tau)$ in Fig. 6. Apart from the effect of statistical errors, CTQMC provides an exact self-consistent solution of DMFT on the imaginary-frequency axis. As mentioned above, when we use the FTPS solver, we formulate the DMFT self-consistency equations on the real axis. To obtain an approximate finite-temperature imaginary-time Green's function from FTPS that we can compare to the CTQMC result, we need to take the finite temperature of the CTQMC calculation into account. Therefore, we use the FTPS spectrum $A(\omega)$ and assume that we would obtain the same spectrum for a finite (but high enough) inverse temperature $\beta$; we use

$$
G(\tau)=\int \frac{d \omega}{2 \pi} A(\omega) \frac{e^{-\omega \tau}}{e^{-\beta \omega}+1} .
$$

The results in Fig. 6 show very good agreement on a logarithmic scale.

Another important indication of the validity of our results is the value of $A(\omega=0)$. To get a comparable number, we use the CTQMC imaginary-time Green's function $G(\tau)$ and Fourier transform it to get $G\left(i \omega_{n}\right)$ :

$$
G\left(i \omega_{n}\right)=\int e^{i \omega_{n} \tau} G(\tau) d \tau
$$

Looking at the last few DMFT cycles, we estimate it to be around $A(\omega=0)=-(1 / \pi) \lim _{i \omega_{n} \rightarrow 0} \Im G\left(i \omega_{n}\right) \approx$ $0.272 \mathrm{eV}^{-1}$, with fluctuations in the last digit. For the FTPS, the exact height of $A(\omega=0)$ of the FTPS spectrum changes a little for each DMFT iteration, mainly due to slight variations in the linear prediction. Using the same prescription as for CTQMC, we estimate it to be $A(\omega=0)=0.28 \mathrm{eV}^{-1}$, with fluctuations of about $0.01 \mathrm{eV}^{-1}$. This agreement is very good considering that linear prediction has its strongest influence at small

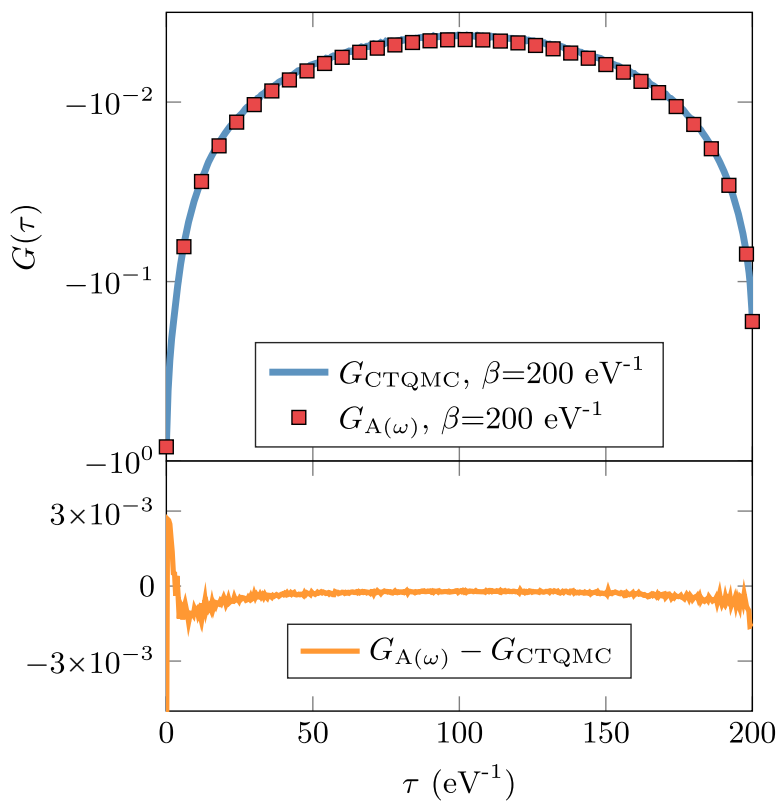

FIG. 6. Comparison of imaginary-time Green's functions $G(\tau)$ from CTQMC ( $\mathrm{G}_{\mathrm{CTQMC}}$, blue line) and FTPS using Eq. (15) $\left(\mathrm{G}_{\mathrm{A}(\omega)}\right.$, red squares). The agreement is also equally good at $\beta=$ $100 \mathrm{eV}^{-1}$ and $\beta=400 \mathrm{eV}^{-1}$ (not shown). The difference between the two Green's functions is shown in the bottom panel. Note that on both ends, $\mathrm{G}_{\mathrm{A}(\omega)}$ is smaller than $\mathrm{G}_{\mathrm{CTQMC}}$. The normalization of the spectral function demands that $G(\tau=0)+G(\tau=\beta)=-1$. The CTQMC data deviate in the order of $10^{-2}$ from this constraint due to statistical noise, while FTPS gives (by construction) the correct result to a precision of $10^{-8}$. This explains the bigger differences of the Green's functions around $\tau=0$ and $\tau=\beta$. For better visibility of the $\tau>0$ data, the value of $9 \times 10^{-3}$ at $\tau=0$ is not shown.

energies. Further benchmarks concerning the linear prediction can be found in Appendix A 1.

Finally, we show that the ill-posedness of the analytic continuation is the most likely explanation for the missing

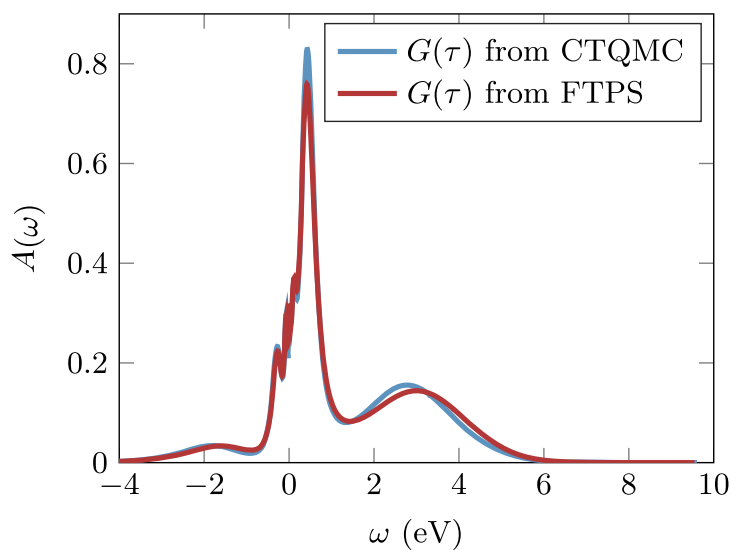

FIG. 7. Spectral functions from analytically continued imaginary-time Green's functions $G(\tau)$ calculated by CTQMC (blue line) and by FTPS (red line). Clearly, the analytic continuation cannot resolve the peak structure in the upper Hubbard band. 
TABLE I. Relevant states of the atomic problem of Hamiltonian (1) without spin-flip and pair-hopping terms.

\begin{tabular}{lccc}
\hline \hline Type & \multicolumn{1}{c}{ States } & Energy difference to ground state & Degeneracy \\
\hline$N=1$, ground state & $|\uparrow, 0,0\rangle|\downarrow, 0,0\rangle|0, \uparrow, 0\rangle \cdots$ & 0 & 6 \\
$N=0$ & $|0,0,0\rangle$ & $-\epsilon_{0}$ & 1 \\
$N=2$, same spin & $|\uparrow, \uparrow, 0\rangle\langle\uparrow, 0, \uparrow\rangle|0, \uparrow, \uparrow\rangle \cdots$ & $U-3 J+\epsilon_{0}$ & 6 \\
$N=2$, different spin & $|\uparrow, \downarrow, 0\rangle|\uparrow, 0, \downarrow\rangle|\downarrow, \uparrow, 0\rangle \cdots$ & $U-2 J+\epsilon_{0}$ & 6 \\
$N=2$, double occupation & $|\uparrow \downarrow, 0,0\rangle|0, \uparrow \downarrow, 0\rangle|0,0, \uparrow \downarrow\rangle$ & $U+\epsilon_{0}$ & 3 \\
$N=3$, all spins equal & $|\uparrow, \uparrow, \uparrow\rangle\langle\downarrow, \downarrow, \downarrow\rangle$ & $3 U-9 J+2 \epsilon_{0}$ & 6 \\
$N=3$, one spin different & $|\uparrow, \uparrow, \downarrow\rangle \uparrow, \downarrow, \uparrow\rangle|\downarrow, \uparrow, \uparrow\rangle$ & $3 U-7 J+2 \epsilon_{0}$ & 12 \\
$N=3$, double occupation & $|\uparrow \downarrow, \uparrow, 0\rangle|\uparrow \downarrow, \downarrow, 0\rangle|\uparrow \downarrow, 0, \uparrow\rangle \cdots$ & $3 U-5 J+2 \epsilon_{0}$ & \\
\hline \hline
\end{tabular}

peak structure in the upper Hubbard band of the spectral function obtained from the CTQMC data. To do so, we take the FTPS spectrum $A(\omega)$, calculate $G(\tau)$ as described above, and perform the same analytic continuation that we did for the $G(\tau)$ from CTQMC. We added noise of the order of the CTQMC error to the FTPS data. The resulting spectrum is shown in Fig. 7, and indeed, the peak structure in the upper Hubbard band vanishes.

\section{Discussion of peak structure-Effective atomic physics}

In order to understand the peak structure observed in the spectral functions, we take a look at the underlying atomic problem, where, for simplicity, we start with densitydensity interactions only. We show that the same arguments hold for full rotationally invariant interactions.

Table I shows the relevant atomic states and their corresponding energies. The atomic model has a hole excitation at energy $-\epsilon_{0}$ and three single electron excitations with energies $U+\epsilon_{0}, U-2 J+\epsilon_{0}$ and $U-3 J+\epsilon_{0}$ relative to the ground state. If we measure the energy differences between the three peaks of the upper Hubbard band in our results, we find values of $1.27 \mathrm{eV}$ and $0.69 \mathrm{eV}$, which are close to the atomic energy differences of $1.2 \mathrm{eV}$ and $0.6 \mathrm{eV}(\mathrm{J}=0.6 \mathrm{eV})$. We also find the hole excitation at $-2.0 \mathrm{eV}$. This indicates that we can describe the positions of the observed peaks approximately by atomic physics with effective parameters $\bar{\epsilon}_{0}, \bar{U}$, and $\bar{J}$ and widened peaks. Furthermore, the heights of the peaks roughly correspond to the degeneracy of the states in the atomic model (see Table I).

We can determine $\bar{U}=5.97 \mathrm{eV}$ (where $U=4.00 \mathrm{eV}$ ) from the energy difference of the peak highest in energy to the hole excitation. This increase of $\bar{U}$ compared to $U$ is plausible, considering the following. When coupling the impurity to the bath, particles have the possibility to avoid each other by jumping into unoccupied sites of the bath. This results in a decrease of $\left\langle n_{\uparrow} n_{\downarrow}\right\rangle$. To model this situation using atomic physics, one needs to increase the interaction strength. Finally, it is well known that $J$ is much less affected by the surrounding electrons than $U$ since the latter is screened significantly stronger [78].
Table II shows how bare atomic parameters change when adding a bath, and we see that our qualitative arguments give a correct idea of how parameters are rescaled.

Further evidence that the observed three-peak structure is indeed a result of atomic physics can be seen in Fig. 8. This figure shows a close-up of the upper Hubbard band for three different values of $J$. The corresponding effective parameters $\bar{J}$ are shown in Table II. We observe that $J$ is also rescaled slightly, but the rescaling gets smaller for higher $J$. Furthermore, increasing $J$ also increases the total width of the Hubbard band, which scales mostly linearly with $J$. At the same time, measuring the quasiparticle spectral weight as a function of $J$ at constant $U$ shows that it increases with increasing $J$, also implying an increasing critical $U_{c}$ for the metal-to-insulator transition [40].

Upon a careful inspection of the spectral function in Fig. 5, we observe small peaks at energies around $8 \mathrm{eV}$. A close-up of this energy region for different values of $J$ is shown in Fig. 8. The energy difference between the peaks is close to $2 J$ and can, again, be well explained by atomic physics, namely, excitations into states with three electrons on the impurity (Table I) [79]. These excitations originate from small admixtures of $N=2$ states to the ground state.

With atomic physics in mind, let us take a look again at the spectrum using full rotational symmetry (Fig. 3). The spin-flip and pair-hopping terms only contribute if there are two or more particles present. Thus, the quasiparticle peak and the hole excitation do not change. The atomic $N=2$ sector does change, however. Diagonalizing the

TABLE II. Atomic parameters and their effective values obtained from the spectral functions shown in Figs. 5 and 8. For $J$, the values themselves were obtained from the energy difference of the highest peak to the lowest peak, whereas the uncertainty is estimated from $\omega_{M}+2 J$ and $\omega_{M}-J$.

\begin{tabular}{lcc}
\hline \hline Parameter & Atomic value $(\mathrm{eV})$ & Effective value $(\mathrm{eV})$ \\
\hline$\epsilon_{0}$ & -0.86 & -2.00 \\
$U$ & 4.00 & 5.97 \\
$J$ & 0.50 & $0.59(6)$ \\
$J$ & 0.60 & $0.66(3)$ \\
$J$ & 0.70 & $0.72(2)$ \\
\hline \hline
\end{tabular}



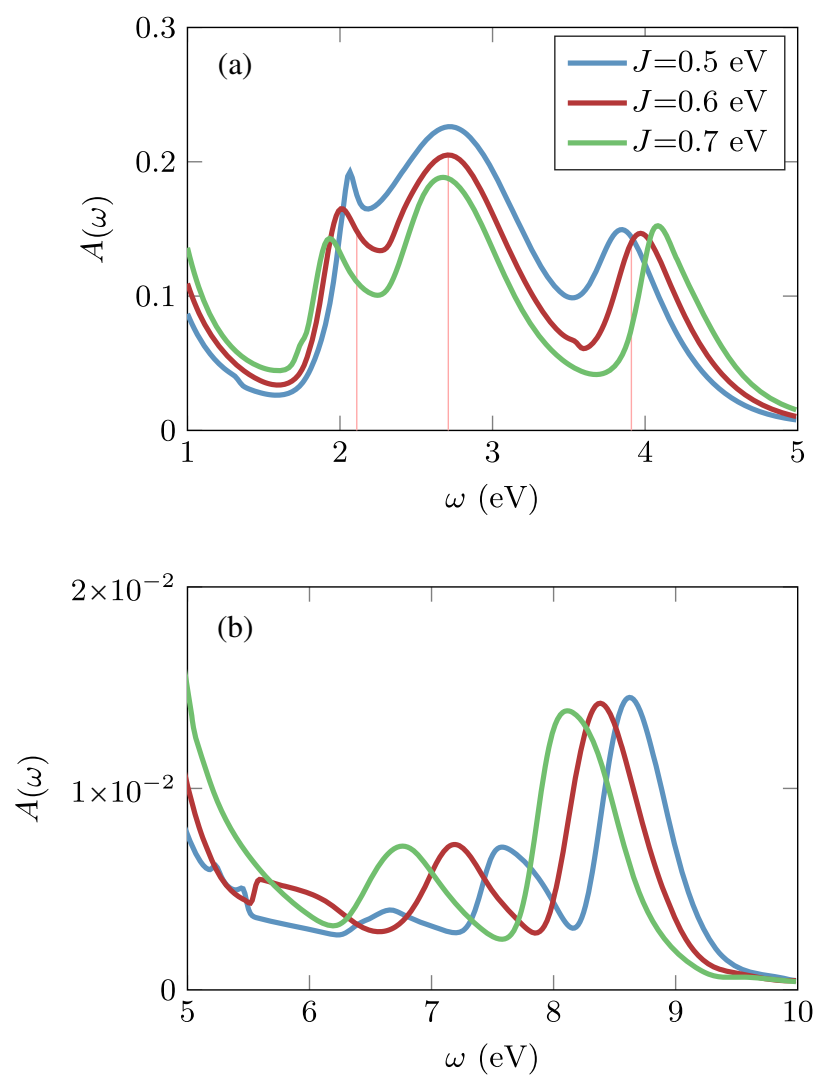

FIG. 8. (a) Close-up of the three-peak structure for various values of $J$. Additionally, we show vertical lines for the $J=$ $0.6 \mathrm{eV}$ spectrum at energies $\omega_{M}$ (position of the middle peak) and at $\omega_{M}+2 J$ and $\omega_{M}-J$. We see that the width of the upper Hubbard band is close to $3 J$. (b) Close-up of the small spectral peaks at high energies. These correspond to excitations into the $N=3$ sector of the atomic model (see Table I). The height of each peak can be estimated by the degeneracy of the atomic states. Effective parameters $\bar{J}$ are $0.53 \mathrm{eV}(J=0.5 \mathrm{eV}), 0.59 \mathrm{eV}$ $(J=0.6 \mathrm{eV})$, and $0.68 \mathrm{eV}(J=0.7 \mathrm{eV})$. They are obtained from the difference between the two peaks that are highest in energy.

Hamiltonian, we find eigenstates with three different energies and differences of $3 J=1.8 \mathrm{eV}$ and $2 J=$ $1.2 \mathrm{eV}$, respectively. Measuring the energy differences in Fig. 3 , we find $3 \bar{J}=1.75 \mathrm{eV}$ and $2 \bar{J}=1.32 \mathrm{eV}$. Estimating $\bar{U}=5.81(5)$, we see that it does not change much compared to DD only [80]. Again, we can describe the spectrum approximately by atomic physics with effective parameters. Like in the case with density-density terms only, we also see the tiny excitations to states belonging to the atomic $N=3$ sector.

\section{Beyond atomic physics}

The previous section showed that at $U=4.0 \mathrm{eV}$, the spectral features in the Hubbard bands can be well described by atomic physics with effective parameters and widened peaks. It is not clear whether this picture is valid for higher interaction strengths $U$ in the metallic regime. In Fig. 9, we show results with $U=5.5 \mathrm{eV}$ at constant $J=0.6 \mathrm{eV}$. We see a shift of the upper Hubbard band to higher energies, but little shift of the hole excitation. Also, the central peak is shifted and gets slimmer since more weight is transferred into the Hubbard bands. Most importantly, as we approach the strongly correlated metallic regime, we clearly leave the realm where atomic physics can describe all the spectral features.

We find that the three-peak structure of the upper Hubbard bands smears out and even vanishes. The close-up of the upper Hubbard band in Fig. 9 shows that with the help of the bare energy differences, all three atomic peaks can be discerned again, accompanied by an additional structure at the low-energy side of the Hubbard band, which is reminiscent of the Hubbard side peaks found in the one- and two-band Hubbard models on the Bethe lattice [35] upon increasing $U$. We leave further investigation of this feature to future work.
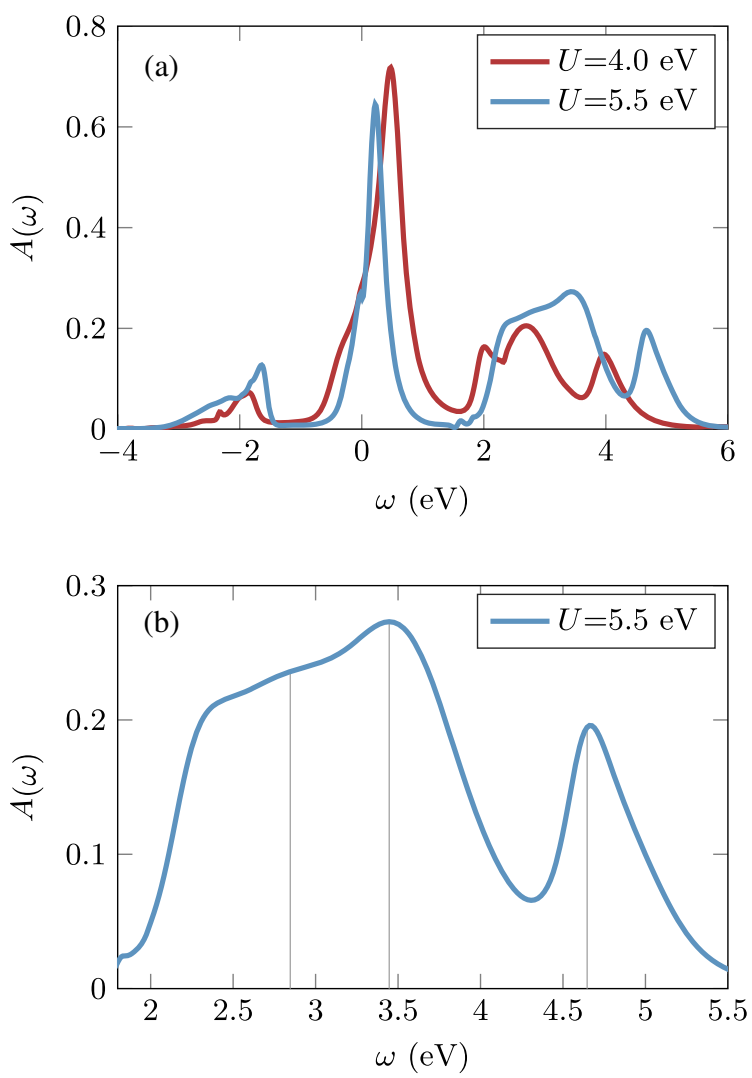

FIG. 9. (a) Increasing $U$ results in a slimmer central peak and a shift of the upper Hubbard band. Also, the three-peak structure gets smeared out. (b) Close-up of the upper Hubbard band. As in Fig. 8, additional vertical lines are plotted at $\omega_{M}$ (position of the middle peak) and at $\omega_{M}+2 J$ and $\omega_{M}-J$ as a rough guide to where the atomic peaks would be located. With the help of these lines, one can discern a three-peak structure again, but it is extended by a feature at the low energy side of the Hubbard band. 
It might at first seem counterintuitive that increasing $U$ makes the physics less atomiclike. Indeed, at very high interaction strengths, in the insulating regime, the spectrum must become atomiclike again. Here, however, we identify an intermediate regime where additional structures appear when increasing $U$ since we get closer to the Mott metal-toinsulator transition.

\section{E. Solution of a five-band AIM}

In this section, we show that FTPS not only applies to three-band models, but it also works in the case of five orbitals. To do so, we use the bath parameters $\epsilon_{k}$ and $V_{k}$ from the converged $N_{b}=59$ calculation for $\mathrm{SrVO}_{3}$ and construct an artificial degenerate five-band AIM. Interaction parameters are $U=4.0 \mathrm{eV}$ and $J=0.6 \mathrm{eV}$. We decrease the on-site energy $\epsilon_{0}$ to get a similar occupation of each impurity orbital as for $\mathrm{SrVO}_{3}$ $\left(\left\langle n_{m, 0, \sigma}\right\rangle \approx \frac{1}{6}\right)$. Note that, in doing so, we have a model with, in total, $\frac{5}{3}$ electrons on the impurity. We only use density-density interactions and carry out the time evolution to $t=16 \mathrm{eV}^{-1}$. We set the truncated weight to $t_{w}=10^{-8}$, but we restrict the bond dimension of the impurity-impurity links to $\chi_{\max }=200$.

In Fig. 10, we compare the results obtained for this fiveband model to results from CTQMC, where we used the same discretized bath hybridization as input to CTQMC. We again see excellent agreement, even on a logarithmic scale. The spectrum $A(\omega)$ (not shown) again exhibits strong structure in the upper Hubbard band. Of course, the computational complexity is larger than in the three-orbital case, and it grows during time evolution. Calculating the

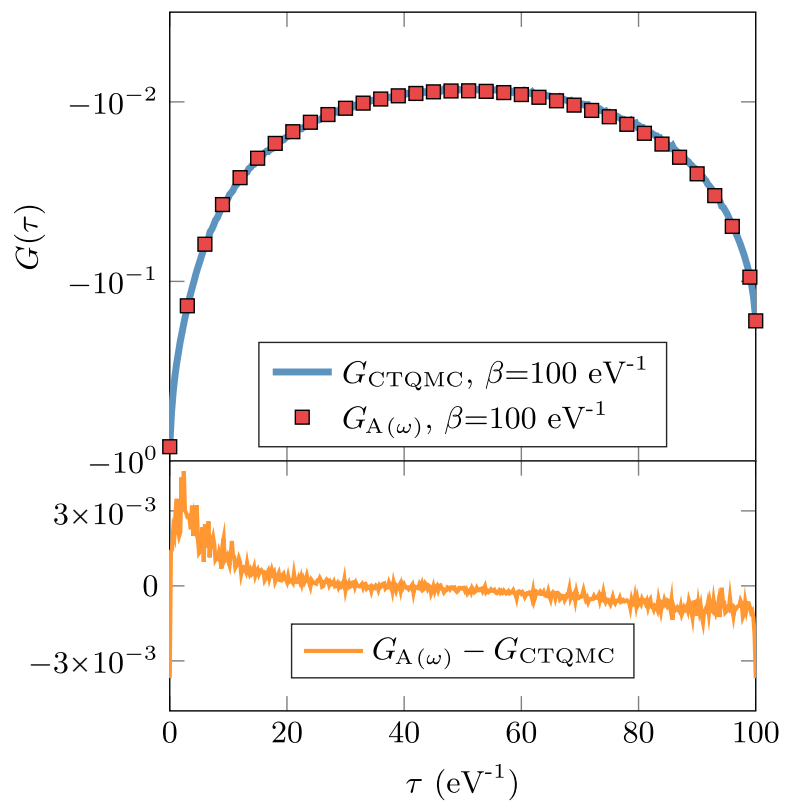

FIG. 10. Comparison of imaginary-time Green's functions $G(\tau)$ from CTQMC $\left(\mathrm{G}_{\mathrm{CTQMC}}\right.$, blue line) and FTPS using Eq. (15) $\left(\mathrm{G}_{\mathrm{A}(\omega)}\right.$, red squares). As in Fig. 6, they compare very well.
Green's function took about 190 hours on the processors specified in Appendix A 2. We want to stress, though, that the resulting spectrum (as well as Fig. 10) was already fully converged at $t=12 \mathrm{eV}^{-1}$ (70 hours). We note that even with only one CPU hour $\left(t=6 \mathrm{eV}^{-1}\right)$, the resulting spectrum is almost converged and barely distinguishable from the final result. The benchmark therefore shows that with our FTPS approach, a full five-orbital DMFT calculation is well within reach.

\section{CONCLUSIONS}

We have presented a novel multi-orbital impurity solver which uses a forklike tensor network whose geometry resembles that of the Hamiltonian. The network structure is simple enough to generate Schmidt decompositions, allowing us to truncate the tensor network safely and to use established methods like DMRG and real-time evolution. The solver works on the real frequency axis and hence allows us to formulate the full DMFT self-consistency procedure for real frequencies. Therefore, results are not plagued by an ill-conditioned analytic continuation. Our approach exhibits no sign problem, though it does become more involved for larger numbers of orbitals.

We tested the solver within DMFT on a Hamiltonian typically used for the test-bed material $\mathrm{SrVO}_{3}$ and investigated the influence of full rotational invariance on the results. We found clear spectral structures, in particular, in the upper Hubbard band that have not been accessible by CTQMC, for which the necessary analytic continuation prohibits the resolution of fine structures in the spectral function at higher energies. For our calculations with $U=4.0 \mathrm{eV}$, each peak in the spectrum corresponds to an atomic excitation. Even excitations into states with three particles on the impurity are resolved, as tiny spectral peaks at high energies. Furthermore, upon increasing $U$, an additional structure appears on the inside of the Hubbard bands, similar to the precursors of the sharp Hubbard side peaks found for the one- and two-band Hubbard models on the Bethe lattice [29,35]. We have also shown that our approach is feasible for five-orbital models, by comparing results from the FTPS solver to CTQMC for an artificial five-band model.

\section{ACKNOWLEDGMENTS}

The authors acknowledge financial support by the Austrian Science Fund (FWF) through SFB ViCoM F41 (P04 and P03), through Project No. P26220, and through the START Program No. Y746, as well as by NAWI-Graz. This research was supported in part by the National Science Foundation under Grant No. NSF PHY-1125915. We are grateful for stimulating discussions with F. Verstraete, K. Held, F. Maislinger, and G. Kraberger. The computational resources have been provided by the Vienna Scientific 
Cluster (VSC). All calculations involving tensor networks were performed using the ITensor library [81].

\section{APPENDIX A: INFLUENCE OF COMPUTATIONAL PARAMETERS}

In this appendix, we show that our results are very stable over a wide range of computational parameters. First, we focus on the linear-prediction method (Sec. A 1). Then, we show that the results are converged with respect to the usual MPS approximation (Sec. A 2).

\section{Linear prediction}

In order to obtain smooth and sharp spectra, we used linear prediction to extrapolate the Green's function in time [28,35]. Without going into detail, we state the fact that linear prediction has two parameters, the pseudo-inverse cutoff $p_{\text {inv }}$ and the order $N$ of the linear prediction. Figure 11 shows that the results are converged in these parameters.

We also show a DMFT run without any linear prediction, which is only possible if we increase the broadening parameter of the Fourier transform to $\eta_{\mathrm{FT}}=0.1 \mathrm{eV}$ since otherwise we would get oscillations due to the hard cutoff of the time series. Except for a shift towards the right, omitting the linear prediction only changes the height (and width) of the peaks but not the overall structure. This is a strong indicator of the stability of these features.

\section{Truncation of the tensor network}

One of the most important parameters in any MPS-like calculation is the sum of discarded singular values in each SVD (truncated weight $t_{w}$ ). We want to emphasize that this

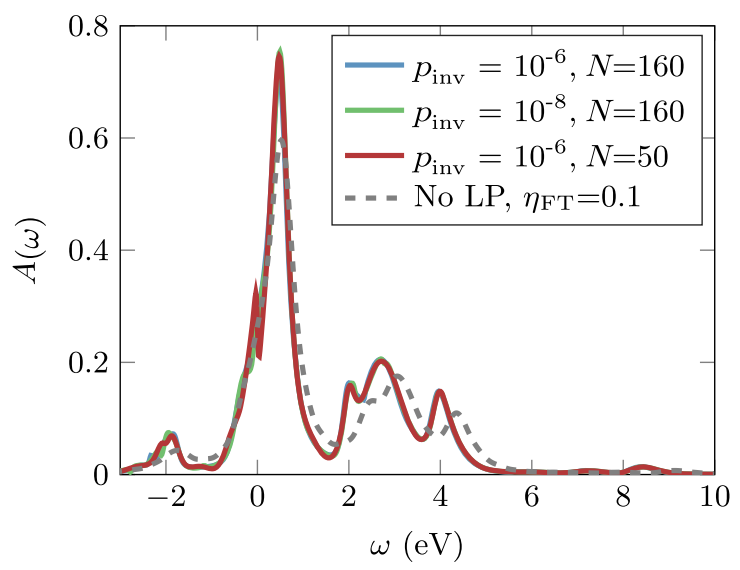

FIG. 11. Spectrum $A(\omega)$ using different linear prediction parameters for a calculation without spin-flip and pair-hopping terms. The calculations with linear prediction were performed with a broadening of $\eta_{\mathrm{FT}}=0.02 \mathrm{eV}$. Except for small changes around $\omega=0$, the effect of the various linear prediction parameters is minor. The blue line lies directly below the red and green lines. We also show a DMFT calculation without any linear prediction. The main features are still present.

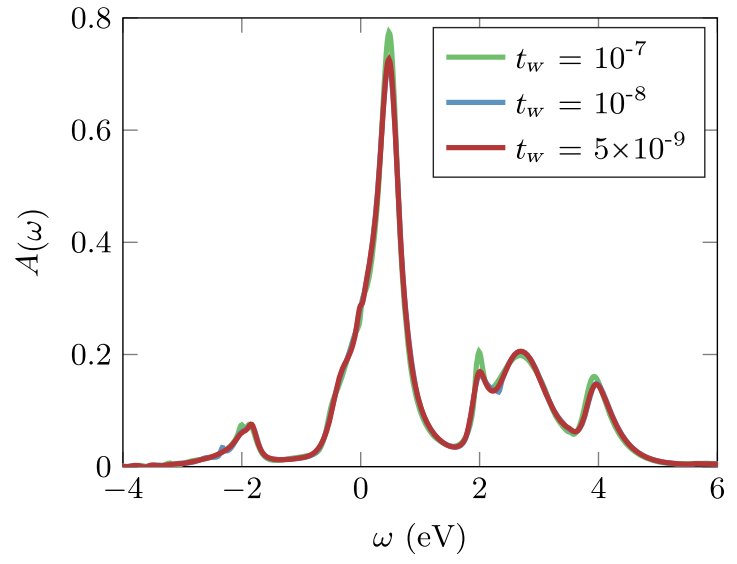

FIG. 12. Different truncation values $t_{w}$ in the time evolution do not influence the shape of the spectrum $A(\omega)$.

parameter is the only approximation in the representation of a state as a tensor-product state, as we do not impose any hard cutoff on the bond dimensions. Figure 12 shows that the spectrum is well converged with respect to the truncation error during time evolution.

Finally, we comment on the required computational effort. In the calculation without full rotational symmetry, the size of the largest tensor to represent the ground state was $35 \times 22 \times 9 \times 2\left(t_{w}=10^{-9}\right)$ [82] and at the end of time evolution $127 \times 79 \times 30 \times 2\left(t_{w}=10^{-8}\right)$. For a truncated weight of $t_{w}=10^{-7}$, calculating the Green's function took about 17 minutes on a node with two processors (Intel Xeon E5-2650v2, $2.6 \mathrm{GHz}$ with eight cores, and $G^{>}$and $G^{<}$each calculated on one processor). This time increases to five hours for the lowest truncated weight of $t_{w}=5 \times 10^{-9}$. Using the full rotationally invariant Hamiltonian, the biggest tensor in the ground-state search was $90 \times 40 \times 10 \times 2\left(t_{w}=10^{-8}\right)$, and at the end of time evolution, $79 \times 46 \times 21 \times 2\left(t_{w}=2 \times 10^{-8}\right)$. The Green's function takes about three hours, and we need five hours for one full DMFT cycle on the same two processors as above.

[1] P. W. Anderson, More Is Different, Science 177, 393 (1972).

[2] A. Georges, G. Kotliar, W. Krauth, and M. J. Rozenberg, Dynamical Mean-Field Theory of Strongly Correlated Fermion Systems and the Limit of Infinite Dimensions, Rev. Mod. Phys. 68, 13 (1996).

[3] W. Metzner and D. Vollhardt, Correlated Lattice Fermions in $d=\infty$ Dimensions, Phys. Rev. Lett. 62, 324 (1989).

[4] E. Gull, A. J. Millis, A. I. Lichtenstein, A. N. Rubtsov, M. Troyer, and P. Werner, Continuous-Time Monte Carlo Methods for Quantum Impurity Models, Rev. Mod. Phys. 83, 349 (2011).

[5] P. Werner, A. Comanac, L. de' Medici, M. Troyer, and A. J. Millis, Continuous-Time Solver for Quantum Impurity Models, Phys. Rev. Lett. 97, 076405 (2006). 
[6] M. Caffarel and W. Krauth, Exact Diagonalization Approach to Correlated Fermions in Infinite Dimensions: Mott Transition and Superconductivity, Phys. Rev. Lett. 72, 1545 (1994).

[7] M. Capone, L. de' Medici, and A. Georges, Solving the Dynamical Mean-Field Theory at Very Low Temperatures Using the Lanczos Exact Diagonalization, Phys. Rev. B 76, 245116 (2007).

[8] J. Kolorenč, A. B. Shick, and A. I. Lichtenstein, Electronic Structure and Core-Level Spectra of Light Actinide Dioxides in the Dynamical Mean-Field Theory, Phys. Rev. B 92, 085125 (2015).

[9] K. G. Wilson, The Renormalization Group: Critical Phenomena and the Kondo Problem, Rev. Mod. Phys. 47, 773 (1975).

[10] R. Bulla, T. A. Costi, and T. Pruschke, Numerical Renormalization Group Method for Quantum Impurity Systems, Rev. Mod. Phys. 80, 395 (2008).

[11] Y. Lu, M. Höppner, O. Gunnarsson, and M. W. Haverkort, Efficient Real-Frequency Solver for Dynamical Mean-Field Theory, Phys. Rev. B 90, 085102 (2014).

[12] D. Zgid, E. Gull, and G. K. L. Chan, Truncated Configuration Interaction Expansions as Solvers for Correlated Quantum Impurity Models and Dynamical Mean-Field Theory, Phys. Rev. B 86, 165128 (2012).

[13] S. R. White, Density Matrix Formulation for Quantum Renormalization Groups, Phys. Rev. Lett. 69, 2863 (1992).

[14] U. Schollwöck, The Density-Matrix Renormalization Group in the Age of Matrix Product States, Ann. Phys. (Amsterdam) 326, 96 (2011).

[15] K. M. Stadler, Z. P. Yin, J. von Delft, G. Kotliar, and A. Weichselbaum, Dynamical Mean-Field Theory Plus Numerical Renormalization-Group Study of Spin-Orbital Separation in a Three-Band Hund Metal, Phys. Rev. Lett. 115, 136401 (2015).

[16] A. Horvat, R. Žitko, and J. Mravlje, Low-Energy Physics of Three-Orbital Impurity Model with Kanamori Interaction, Phys. Rev. B 94, 165140 (2016).

[17] K. M. Stadler, A. K. Mitchell, J. von Delft, and A. Weichselbaum, Interleaved Numerical Renormalization Group as an Efficient Multiband Impurity Solver, Phys. Rev. B 93, 235101 (2016).

[18] K. A. Hallberg, Density-Matrix Algorithm for the Calculation of Dynamical Properties of Low-Dimensional Systems, Phys. Rev. B 52, R9827 (1995).

[19] D. J. García, K. Hallberg, and M. J. Rozenberg, Dynamical Mean Field Theory with the Density Matrix Renormalization Group, Phys. Rev. Lett. 93, 246403 (2004).

[20] T. D. Kühner and S. R. White, Dynamical Correlation Functions Using the Density Matrix Renormalization Group, Phys. Rev. B 60, 335 (1999).

[21] E. Jeckelmann, Dynamical Density-Matrix Renormalization-Group Method, Phys. Rev. B 66, 045114 (2002).

[22] S. Nishimoto and E. Jeckelmann, Density-Matrix Renormalization Group Approach to Quantum Impurity Problems, J. Phys. Condens. Matter 16, 613 (2004).

[23] R. Peters, Spectral Functions for Single- and Multi-impurity Models Using Density Matrix Renormalization Group, Phys. Rev. B 84, 075139 (2011).
[24] M. Karski, C. Raas, and G. S. Uhrig, Single-Particle Dynamics in the Vicinity of the Mott-Hubbard Metal-toInsulator Transition, Phys. Rev. B 77, 075116 (2008).

[25] M. Karski, C. Raas, and G. S. Uhrig, Electron Spectra Close to a Metal-to-Insulator Transition, Phys. Rev. B 72, 113110 (2005).

[26] A. Weiße, G. Wellein, A. Alvermann, and H. Fehske, The Kernel Polynomial Method, Rev. Mod. Phys. 78, 275 (2006).

[27] A. Holzner, A. Weichselbaum, I. P. McCulloch, U. Schollwöck, and J. von Delft, Chebyshev Matrix Product State Approach for Spectral Functions, Phys. Rev. B 83, 195115 (2011).

[28] S. R. White and I. Affleck, Spectral Function for the $S=1$ Heisenberg Antiferromagnetic Chain, Phys. Rev. B 77, 134437 (2008).

[29] M. Ganahl, P. Thunström, F. Verstraete, K. Held, and H. G. Evertz, Chebyshev Expansion for Impurity Models Using Matrix Product States, Phys. Rev. B 90, 045144 (2014).

[30] F. A. Wolf, I. P. McCulloch, O. Parcollet, and U. Schollwöck, Chebyshev Matrix Product State Impurity Solver for Dynamical Mean-Field Theory, Phys. Rev. B 90, 115124 (2014).

[31] G. Vidal, Efficient Classical Simulation of Slightly Entangled Quantum Computations, Phys. Rev. Lett. 91, 147902 (2003).

[32] G. Vidal, Efficient Simulation of One-Dimensional Quantum Many-Body Systems, Phys. Rev. Lett. 93, 040502 (2004).

[33] S. R. White and A. E. Feiguin, Real-Time Evolution Using the Density Matrix Renormalization Group, Phys. Rev. Lett. 93, 076401 (2004).

[34] A. J. Daley, C. Kollath, U. Schollwöck, and G. Vidal, Time-Dependent Density-Matrix Renormalization-Group Using Adaptive Effective Hilbert Spaces, J. Stat. Mech. (2004) P04005.

[35] M. Ganahl, M. Aichhorn, H. G. Evertz, P. Thunström, K. Held, and F. Verstraete, Efficient DMFT Impurity Solver Using Real-Time Dynamics with Matrix Product States, Phys. Rev. B 92, 155132 (2015).

[36] F. A. Wolf, A. Go, I. P. McCulloch, A. J. Millis, and U. Schollwöck, Imaginary-Time Matrix Product State Impurity Solver for Dynamical Mean-Field Theory, Phys. Rev. X 5, 041032 (2015).

[37] A. Holzner, A. Weichselbaum, and J. von Delft, Matrix Product State Approach for a Two-Lead Multilevel Anderson Impurity Model, Phys. Rev. B 81, 125126 (2010).

[38] P. Werner, E. Gull, M. Troyer, and A. J. Millis, Spin Freezing Transition and Non-Fermi-Liquid Self-Energy in a Three-Orbital Model, Phys. Rev. Lett. 101, 166405 (2008).

[39] L. de' Medici, J. Mravlje, and A. Georges, Janus-Faced Influence of Hund's Rule Coupling in Strongly Correlated Materials, Phys. Rev. Lett. 107, 256401 (2011).

[40] A. Georges, L. de' Medici, and J. Mravlje, Strong Correlations from Hund's Coupling, Annu. Rev. Condens. Matter Phys. 4, 137 (2013).

[41] A. J. Kim, H. O. Jeschke, P. Werner, and R. Valentí, J-Freezing and Hund's Rules in Spin-Orbit-Coupled Multiorbital Hubbard Models, Phys. Rev. Lett. 118, 086401 (2017). 
[42] F. Verstraete and J. I. Cirac, Matrix Product States Represent Ground States Faithfully, Phys. Rev. B 73, 094423 (2006).

[43] V. Murg, F. Verstraete, Ö. Legeza, and R. M. Noack, Simulating Strongly Correlated Quantum Systems with Tree Tensor Networks, Phys. Rev. B 82, 205105 (2010).

[44] F. A. Wolf, I. P. McCulloch, and U. Schollwöck, Solving Nonequilibrium Dynamical Mean-Field Theory Using Matrix Product States, Phys. Rev. B 90, 235131 (2014).

[45] H. Guo and S. R. White, Density Matrix Renormalization Group Algorithms for Y-Junctions, Phys. Rev. B 74, 060401 (2006)

[46] Y. Y. Shi, L. M. Duan, and G. Vidal, Classical Simulation of Quantum Many-Body Systems with a Tree Tensor Network, Phys. Rev. A 74, 022320 (2006).

[47] L. Tagliacozzo, G. Evenbly, and G. Vidal, Simulation of Two-Dimensional Quantum Systems Using a Tree Tensor Network that Exploits the Entropic Area Law, Phys. Rev. B 80, 235127 (2009).

[48] I. Pižorn, F. Verstraete, and R. M. Konik, Tree Tensor Networks and Entanglement Spectra, Phys. Rev. B 88, 195102 (2013).

[49] P. Schmitteckert, Nonequilibrium Electron Transport Using the Density Matrix Renormalization Group Method, Phys. Rev. B 70, 121302 (2004).

[50] J. Haegeman, J. I. Cirac, T. J. Osborne, I. Pižorn, H. Verschelde, and F. Verstraete, Time-Dependent Variational Principle for Quantum Lattices, Phys. Rev. Lett. 107, 070601 (2011).

[51] J. Haegeman, C. Lubich, I. Oseledets, B. Vandereycken, and F. Verstraete, Unifying Time Evolution and Optimization with Matrix Product States, Phys. Rev. B 94, 165116 (2016).

[52] M. P. Zaletel, R. S. K. Mong, C. Karrasch, J. E. Moore, and F. Pollmann, Time-Evolving a Matrix Product State with Long-Ranged Interactions, Phys. Rev. B 91, 165112 (2015).

[53] M. Suzuki, Fractal Decomposition of Exponential Operators with Applications to Many-Body Theories and Monte Carlo Simulations, Phys. Lett. A 146, 319 (1990).

[54] Note that we use the term MPO a bit loosely here. What we are referring to is an operator factorized in the same forklike structure as the state in Fig. 2.

[55] E. M. Stoudenmire and S. R. White, Minimally Entangled Typical Thermal State Algorithms, New J. Phys. 12, 055026 (2010).

[56] A. Georges, Strongly Correlated Electron Materials: Dynamical Mean-Field Theory and Electronic Structure, in American Institute of Physics Conference Series, edited by A. Avella and F. Mancini (AIP, New York, 2004), Vol. 715, pp. 3-74.

[57] F. Lechermann, A. Georges, A. Poteryaev, S. Biermann, M. Posternak, A. Yamasaki, and O. K. Andersen, Dynamical Mean-Field Theory Using Wannier Functions: A Flexible Route to Electronic Structure Calculations of Strongly Correlated Materials, Phys. Rev. B 74, 125120 (2006).

[58] G. Kotliar, S. Y. Savrasov, K. Haule, V. S. Oudovenko, O. Parcollet, and C.A. Marianetti, Electronic Structure Calculations with Dynamical Mean-Field Theory, Rev. Mod. Phys. 78, 865 (2006).
[59] For stability reasons, a larger broadening of $\eta_{\mathrm{SC}}=0.01 \mathrm{eV}$ was used in the first two DMFT cycles.

[60] E. Koch, G. Sangiovanni, and O. Gunnarsson, Sum Rules and Bath Parametrization for Quantum Cluster Theories, Phys. Rev. B 78, 115102 (2008).

[61] I. de Vega, U. Schollwöck, and F. A. Wolf, How to Discretize a Quantum Bath for Real-Time Evolution, Phys. Rev. B 92, 155126 (2015).

[62] J. G. Liu, D. Wang, and Q. H. Wang, Quantum Impurities in Channel Mixing Baths, Phys. Rev. B 93, 035102 (2016).

[63] T. Barthel, Precise Evaluation of Thermal Response Functions by Optimized Density Matrix Renormalization Group Schemes, New J. Phys. 15, 073010 (2013).

[64] Indeed, model calculations performed for fillings of $N=2$ and $N=3$ electrons (the latter in the insulating phase) show that these calculations are of comparable computational effort. For any $N$, we do expect increased numerical effort close to the Mott transition though.

[65] P. Blaha, K. Schwarz, G. Madsen, D. Kvasnicka, and J. Luitz, WIEN2k, An Augmented Plane Wave +Local Orbitals Program for Calculating Crystal Properties (Techn. Universitat Wien, Austria, 2001).

[66] M. Aichhorn, L. Pourovskii, P. Seth, V. Vildosola, M. Zingl, O. E. Peil, X. Deng, J. Mravlje, G. J. Kraberger, C. Martins, M. Ferrero, and O. Parcollet, TRIQS/DFTTools: A TRIQS Application for Ab Initio Calculations of Correlated Materials, Comput. Phys. Commun. 204, 200 (2016).

[67] M. Aichhorn, L. Pourovskii, V. Vildosola, M. Ferrero, O. Parcollet, T. Miyake, A. Georges, and S. Biermann, Dynamical Mean-Field Theory within an Augmented Plane-Wave Framework: Assessing Electronic Correlations in the Iron Pnictide LaFeAsO, Phys. Rev. B 80, 085101 (2009).

[68] M. Aichhorn, L. Pourovskii, and A. Georges, Importance of Electronic Correlations for Structural and Magnetic Properties of the Iron Pnictide Superconductor LaFeAsO, Phys. Rev. B 84, 054529 (2011).

[69] O. Parcollet, M. Ferrero, T. Ayral, H. Hafermann, I. Krivenko, L. Messio, and P. Seth, TRIQS: A Toolbox for Research on Interacting Quantum Systems, Comput. Phys. Commun. 196, 398 (2015).

[70] A. Liebsch, Surface versus Bulk Coulomb Correlations in Photoemission Spectra of $\mathrm{SrVO}_{3}$ and $\mathrm{CaVO}_{3}$, Phys. Rev. Lett. 90, 096401 (2003).

[71] A. Sekiyama, H. Fujiwara, S. Imada, S. Suga, H. Eisaki, S. I. Uchida, K. Takegahara, H. Harima, Y. Saitoh, I. A. Nekrasov et al., Mutual Experimental and Theoretical Validation of Bulk Photoemission Spectra of $\mathrm{Sr}_{1-x} \mathrm{Ca}_{x} \mathrm{VO}_{3}$, Phys. Rev. Lett. 93, 156402 (2004).

[72] We note that high-energy peaks already appear in the first DMFT iteration, for which the bath does not have any spectral weight at high energies.

[73] G. Sangiovanni, A. Toschi, E. Koch, K. Held, M. Capone, C. Castellani, O. Gunnarsson, S.-K. Mo, J. W. Allen, H.-D. Kim, A. Sekiyama, A. Yamasaki, S. Suga, and P. Metcalf, Static versus Dynamical Mean-Field Theory of Mott Antiferromagnets, Phys. Rev. B 73, 205121 (2006).

[74] E. Gull, D. R. Reichman, and A. J. Millis, Bold-Line Diagrammatic Monte Carlo Method: General Formulation 
and Application to Expansion around the Noncrossing Approximation, Phys. Rev. B 82, 075109 (2010).

[75] P. Seth, I. Krivenko, M. Ferrero, and O. Parcollet, TRIQS/ CTHYB: A Continuous-Time Quantum Monte Carlo Hybridisation Expansion Solver for Quantum Impurity Problems, Comput. Phys. Commun. 200, 274 (2016).

[76] P. Werner and A. J. Millis, Hybridization Expansion Impurity Solver: General Formulation and Application to Kondo Lattice and Two-Orbital Models, Phys. Rev. B 74, 155107 (2006).

[77] D. Bergeron and A.-M.S. Tremblay, Algorithms for Optimized Maximum Entropy and Diagnostic Tools for Analytic Continuation, Phys. Rev. E 94, 023303 (2016).
[78] L. Vaugier, H. Jiang, and S. Biermann, Hubbard U and Hund Exchange J in Transition Metal Oxides: Screening versus Localization Trends from Constrained Random Phase Approximation, Phys. Rev. B 86, 165105 (2012).

[79] Nevertheless, the effective parameters $\bar{J}$ differ a little from those obtained from the main Hubbard band.

[80] Note that the peak highest in energy has an atomic energy of $E=U+2 J+2 \epsilon_{0}$. Therefore, $\bar{U}$ can only be determined after $\bar{J}$ is found.

[81] ITensor library, http://itensor.org.

[82] The numbers 35 and 22 correspond to the impurity links, 9 is the bond dimension to the first bath site, and 2 is the physical bond dimension. 